\title{
JournalScan
}

\section{Relationship between quality, safety and organisational behaviour}

Compiled by Tom Smith

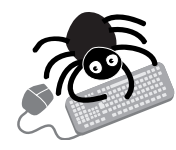

Please visit the website (www.qualityhealthcare.com) for links to these articles - many to full text.

The theme of this review of papers published from September to November 2001 is the relationship between quality, safety, and organisational behaviour. One of the consistent messages in recent NHS policy has been that improving clinical quality to a great extent requires greater synergy between the organisational and clinical domains. A number of journal articles from a range of sources are drawn on to illuminate this relationship from different angles. What information is useful for monitoring quality? How accurate is clinical review information and consequently its suggested implications for clinical practice? Are behavioural or cognitive approaches the most effective route to safe practice? What is known about organisational efforts to improve quality? How can we learn more?

\section{Health information}

US/UK collaboration on health quality data $>$ In October the US and UK Secretaries of State for Health met in Washington. Aside from agreeing to work together to counter bioterrorism, they signed a "joint statement of intent" to collaborate on data and measurement regarding quality of care. "Under the quality agreement, the US and UK plan to share data and experiences. . including efforts to enhance the use of information technology, expand common criteria for measurement of quality of care and achieve mutual quality research goals."

A FDCG Regulatory Intelligence Database. 10 October 2001

A few papers highlight the importance of improving the quality of clinical data, the way it is recorded, reviewed and used.

How accurate is symptom information in medical records and how useful are medical records for assessing the quality of care? Medical records are commonly used to measure quality of care, but how accurate is the documentation and how useful are they as a measure of quality? In mental health, Medicare and Medicaid use records to gather information on the care provided to schizophrenic patients. Recent evidence has highlighted inaccuracies. It is not known whether these findings hold more widely, whether medical records regularly fail to reflect a patient's clinical condition. "Even less is understood about what influences the accuracy of the care provider's documentation and whether patient characteristics impact documentation habits." The paper compares the documented symptoms and side effects in the medical records of 244 patients in two US mental health clinics with those evaluated by direct assessment. It found observed symptoms to be frequently absent from medical records. This has a few implications because "conducting quality assessment, facilitating coordination of care, and managing an individual patient's treatment all can rely on medical records". Some of the reasons for lack of documentation are complex. "Patients who doctors believe are poorly compliant with medication are less likely to have symptoms documented". Patients may not be communicative. And doctors may believe that symptoms will be revealed over time. The paper examines trends in the discrepancies. Documentation was more likely to be absent "for patients who were severely ill, black, or perceived as non-compliant" (76\% of the sample). It suggests future research might focus on the interaction between clinician and patient and, in particular, how the behaviour of the clinician contributes to non-compliance with documentation. It concludes that "the accuracy and consistency of medical record documentation should be demonstrated before using it to evaluate care at public mental health clinics". It is suggested that measuring quality of care should include a variety of sources such as patient interviews and computerised data. "While the quality of documentation and quality of care may be related, they should be treated as separate issues of concern."

$\Delta$ Craddock J, Young A, Sullivan G. The accuracy of medical record documentation in schizophrenia. J Behav Health Serv Res 2001;28:456-66.

The quality of Cochrane reviews - One of the ways of ensuring quality information is through systematic reviews. A paper in the $B M$ written by 10 methodologists suggests they are not infallible. While only minor problems were found with most reviews, "major problems were identified in 15 (29\%). The evidence did not fully support the conclusion in nine reviews $(17 \%)$, the conduct or reporting was unsatisfactory in $12(23 \%)$, and stylistic problems were identified in $12(23 \%)^{\prime \prime}$. Although bias can be a problem in some papers, it is generally less so with Cochrane reviews, although errors and biases may occasionally occur. Too often, reviewers overrate the benefits of new interventions. The team conclude that "users should interpret reviews cautiously, particularly those with conclusions favouring experimental interventions and those with many typographical errors" and state that "no matter what sources of evidence are being used, users of the evidence need to learn the skills of critical appraisal".

$\Delta$ Olsen O, Middleton P, Ezzp J, Gotzsche P, Hadhazy V, Herxheimer A, Kleijnen J, Mclntosh H. Quality of Cochrane reviews: assessment of sample from 1998. BN;2001;323:829-32.

How can health information be verified? While casting doubt on the quality of some information, it is clearly an essential part of health care and its improvement-so much so that new software is being developed all the time to use this information. New technologies also bring new risks. A BM paper highlights examples of "health threatening software errors". The paper reports the findings of a European project towards "accreditation and certification of telematics services in health". It concludes that "public safety and professional integrity are threatened by the lack of regulation of health informatics services". As the volume of information continues to grow, so will the risks. The authors suggest that the cost of developing a system to verify quality would be "high" and "impractical". Instead, they propose a "EuroSeal" approach-kitemarking - whereby a third party would assess a site providers' claims and decide accreditation.

$\Delta$ Rigby M, Forsstrom J, Roberts R, Wyatt J. Verifying quality and safety in health informatics services. BM 2001;323:552-6.

\section{Behaviour and safety}

The role of marketing in promoting safe behaviour $>$ How does important information get through to people? Of the 10 leading causes of death in the United States, at least seven would be reduced substantially if people at risk would change five behaviours. Why don't they? Health education campaigns have raised public awareness "but left behaviour unchanged". Government has found it difficult to get through to the general public. Skin cancer is the world's most prevalent cancer and is preventable. It provides a useful case study for examining information and behaviour change. Only $3 \%$ of children have talked about skin cancer, far less than have talked about smoking and alcohol. The researchers used focus group research (in high risk Tasmania and 
low risk Cardiff) to probe parents' reactions to health information. "Because the aim was to discover participants' normal beliefs and levels of knowledge, they were not informed of the specific topic but were invited to 'a discussion on child health and safety'." The Australians were much more aware, but this knowledge was not reflected in their behaviour. Adults tend to think that targeting adolescents is "difficult" and "hopeless" (including doctors). The implications of the findings include a need for more holistic public policy such as planting trees in open spaces (for shade) or changing school policy. There is also a need to reposition "sun safety" as just "safety". People do not equate sun exposure to tobacco or alcohol. They see it more on a level with "eat your greens" campaigns. The authors argue that skin cancer is an area where social marketing could "do enormous good". Members of focus groups have suggested that organisations such as McDonalds or Coca Cola could help to promote the safety message. This approach focuses on changing behaviour rather than knowledge or attitudes.

A Peattie K, Peattie S, Clarke P. Skin cancer prevention, re-evaluating the public policy implications. J Public Policy Marketing 2001;20:268-80.

What influences safe behaviour? $>$ A parallel theme-the connection between behaviour and attitudes-is discussed in another paper which argues that approaches to safety are based on a psychological model of behaviourism whose limits in changing behaviour are known. Cognitive psychology offers some explanation on why people continue to court risk, such as its understanding of "insufficient justification". On balance, external consequences tend still to favour at risk behaviour. The author suggests that people do not have to experience negative implications to change behaviour. Cognitive psychology-the way thoughts, perceptions, attitudes, and judgements drive behaviour - provides more useful insights into safety. It is impractical to follow the behavioural approach as it depends on constant observation. Safety approaches tend to focus on accidents largely because they are easier to measure. But accident measures do not account for lucky risky behaviour and "are easily biased when reward or punishment is made contingent on them". A more fruitful approach is to focus on internal attitudes rather than external responses to behaviour. There are several ways of exploring these connections: through group membership and "social influence", through probing risk perception, and by managerial attention to safety. "Behavioural safety needs to be dragged into the cognitive era".

\ Kamp J. Cognitive era. Professional Safety 2001;46:30-5.

Safety in amusement parks - Amusement parks depend greatly on the need to be seen as safe. A study of the behaviour of ride operators follows Kamp's call for a cognitive approach to safety. Eighty rides (big, small, and medium) were observed for 30 minutes in eight amusement parks. The causes of incidents are "dynamic and multifaceted, embedded in existing safety cultures and human behaviour". The observers set out to explore at risk behaviour and the extent these relate to individual attention, communication and procedure, the causal factors, and possible corrective measures. On large rides $150 \%$ of amusement park usage) risk is greatest and "crews" are largest. Most at risk behaviour related to procedures, "incorrect dispatching", followed closely by communication. Medium sized rides have a lower accident experience; operated by two or three people, they depend on coordination of roles. The highest proportion of at risk behaviour related to unloading. The small children's rides with sole operators depend greatly on that individual ensuring safety, helping the child onto the ride and securing them in a seat. Most of the problems related to inadequate communication with the child or parent(s). "During the study the observers noted that employee behaviours were different during the first 10 minutes and the last 20 minutes of each sample." It is suggested that 10 minutes was long enough for operators to become accustomed to observation and to revert to behaviour considered normal. By observing patterns in behaviour, at risk behaviour can be reduced by identifying system flaws and root causes that create them. The authors conclude that the "behaviour sampling technique can be an effective means of discovering flaws in systems that need to be fixed or improved".

A Lyon B. Behavior sampling. Professional Safety 2001;46:35-43.
A human approach to safety in healthcare organisations? - An article from a health context suggests a "blunt end/sharp end" model for thinking about patient safety. It is critical of approaches to safety that create a climate of defensiveness and instead suggests that managers should see themselves as a critical part of safety. They are the blunt end-unable to make a direct change-but they can create an environment where those at the sharp end think about the systemic contributors to critical incidents. Managers should use their systemic thinking to draw on the expertise of those at the sharp end to create responsive systems that provide a safety net for good practice. "The reliability of our health care delivery system rests on people, but unfortunately, systems that rely on perfect performance by individuals to prevent errors are doomed to fail. The reason is simple: all humans make mistakes. Physicians and other hospital leaders must understand that only when human mistakes are accepted as inevitable will it be possible to shift away from a punitive frame of mind and focus on identifying underlying systems failures. The traditional approach of fixing blame, imposing discipline, retraining and writing new policies will not prevent human error. It will stifle discussion and discovery of the causes of error."

$\Delta$ White J, Ketring S. True patient safety begins at the top. Physician Exec 2001;27:40-6.

Must accidents happen? "No one has yet learned how to make the inevitable avoidable." This paper draws some insights from "high reliability organisations" (HROs). It takes a systems approach and states that, in any dependent system, a problem in one area snowballs into other areas with which it is "entwined". It is suggested that the key to minimising the risk of accidents is as much about organisation as it is about professional practice. HROs spend "disproportionately more money than other organizations training people to recognize and respond to anomalies". They give them authority and trust them to act in unexpected circumstances. This training is connected to the way people work. The extent to which a team is organised to learn is a key determinant. One of the examples in the paper is a paediatric intensive care unit which is designed to gather knowledge from everyone in the team, recognising that those working with patients have the greatest knowledge to bear. This requires ample opportunity for communication between people who work together. In a healthcare context, the paper questions whether medicine generally has a culture in which open discussion can flourish. Citing the IOM 1999 report on medical error, they consider that many problems result from colleagues not questioning each other. People in clinical teams are not rewarded for questioning the thinking of doctors or others. Until that changes, accidents will happen.

$\Delta$ Roberts K, Bea R, Bartles D. Must accidents happen? Acad Manage Rev $2001 ; 15: 70-80$

\section{Quality in practice}

Equipping teams for learning Edmondson et al have published several papers on learning in teams. This paper reports the results of research with 16 cardiac surgical teams and makes some comments about the ways in which teams learn. Cross-functional teams have become a central part of organisational thinking. "Successful teams must be able to adapt quickly to new ways of working." The study followed the length of time it took each team to incorporate a "difficult new procedure". It also identified a basic philosophy of learning - the more you do something, the better you get at it. Certain teams learn faster than others. The way in which a team is organised-whether it has a design and management for learning -is a strong determinant. It was found that teams which learnt most rapidly had "an environment of psychological safety". Underlying this, and a major element for successful team learning, was a leader who managed learning efforts. The paper concludes that clinical team leaders should not be chosen on the basis of technical ability alone. They must also be capable of creating a learning environment. Paradoxically, this involves leaders working alongside team members and shedding hierarchical status.

A Edmondson A, Bohmer R, Pisano G. Speeding up team learning. Harvard Business Rev 2001;79:125-33. 
Predictors of quality - An observational study of primary care practices also found team working to be an important predictor of quality. From its random sample of 60 practices, four variables stood out as predictors of quality of care: (1) length of consultation; (2) size of practice - while diabetes care was better in larger practices and in practices where staff reported better team climate, access to care was better in small practices; (3) deprivation predicted a poorer uptake of preventative care; and (4) team climate was associated with quality of care for diabetes, good access, continuity of care, and overall satisfaction (this was the only variable that was associated with high quality care across a range of aspects). The study's focus on "team climate" is to explore how people work together and the support required for high quality care. It concludes that "general practice needs effective teamwork"

- Campbell S, Hann M, Hacker J, Burns C, Oliver D, Thapar A, Mead N, Gelb Safran D, Roland M. Identifying predictors of high quality care in English general practice: observational study. BM 2001;323:784.

\section{Management knowledge}

The academic contribution to the downfall of TQM? - What do we know about the role of management in improving quality and safety? Not much, suggests a paper based on an analysis of UK doctoral theses on TQM between 1981 and 1992. There has been little attempt to consolidate what is known. It found "a clear absence of a meta model of TQM that summarizes the 'what' and the 'how' of organizational challenges". A key cause for discrediting TQM is the failure to build a coherent research base. Each thesis draws out new guidelines - possibly because of the onus on "newness" in doctoral research. The paper notes a reluctance to use existing methods to explore experience and build up knowledge. There has been little attempt to build up or on a body of research or to try to build up alternative dimensions-a meta-view-of the phenomenon. It concludes that doctoral research from 1981 to date provides a number of insights for future research opportunities to consolidate managerial knowledge in this area.

$\Delta$ Zain Z, Dale B, Kehoe F. Doctoral TQM research: a study of themes, directions and trends. Total Quality Manage 2001;12:599-70.

TQM and the NHS manager - The kind of research wanted by Zain et al is contained in a paper in the same issue which looks at TQM from the perspective of the NHS manager. In 1990 the Department of Health endorsed TQM as a way to improve health care. But "most NHS managers have lost interest in TQM as a strategy for improving organizational performance because of the general belief that TQM fails to tackle the critical needs of organizations in trouble". It is suggested that the failure of "most TQM programmes is down to complicated methodologies adopted by organizations". TQM has been understood in different ways and competing explanations exist. It makes more sense to take a "common sense" approach that sees TQM as all pervading, relating to strategy, customer satisfaction, efficient practice, and process redesign. Overelaborate prescriptions and competing views fail to address, in the author's opinion, "three key questions that are close to the hearts of employees: 'How does TQM affect me as an individual?'; 'What's in it for me and what is expected of me?'; and, fundamentally, 'Where do I take my problems to'?" The problem is a lack of a meaningful definition. One of the roles of managers is to tailor TQM to local needs and to translate it in practical terms for different roles and the organisation as a whole.

$\Delta$ Nwabueze E. The implementation of TQM for the NHS manager. Total Qual Manage 2001;12:657-76.

Can management and medical science be integrated? $>$ A US paper on the past, present, and future of healthcare quality suggests that the progress of thinking about quality is a progressive entwining of management and medical science. It identifies three paths towards quality health care: regulatory, learning science, and management science. "The regulatory route leads to punishment and blame. The learning science path splits, with one road leading to regulation (standard setting) and the other (experimental approach) to the halls of academic medicine." The authors suggest that management science has insights that can be married to the learning science path. Until relatively recently, the learning science approach dominated concepts in healthcare quality but has come under greater critical scrutiny. The IOM report To Err is Human may have finished it as a discrete approach to quality improvement. The public have had to come to terms with the fact that the same system capable of producing miracles was responsible for "44 000 to 98000 deaths annually in hospitals due to medical errors" - the fifth leading cause of death in the USA. Furthering the learning science standard approach is not the way ahead. "If they judge a provider in non-compliance with standards, they can hold [them] accountable through mandatory recommendations, sanctions, financial penalties, and even imprisonment. This is a far cry from learning. It is fear." The focus of management science on process and continuous quality improvement took hold in the 1990s but "the wave passed by". Among the reasons for the failure of what the author calls the "1987-1995 experience" are: being seen as an invasion by an alien culture, uncomfortable and to be fought off; the business case for quality was never effectively presented; and purchasers and patients have been unable to "distinguish high quality from mediocrity". It is suggested that quality should maintain the best of the learning science tradition, which means detoxifying medical peer review practices and restoring a learning-based peer case review. Some attempt is needed to understand organisational culture and the culture of blame prevalent in hospitals. It is concluded that there is a need to integrate management science with clinical care and care system design and to establish external review processes that can genuinely help committed organisations achieve their goals.

$\Delta$ Merry M, Michael G. The past, present and future of health care quality. Physician Exec 2001;27:30-6.

T Smith

Judge Institute of Managment Studies, Cambridge University, Cambridge CB2 1 1AG, UK; tabletom@btinternet.com 